\title{
Using a Configuration System to Design Toilets and Place Installation Shafts
}

\author{
Anders Kudsk ${ }^{1, *}$, Lars Hvam ${ }^{2}$ and Christian Langhoff Thuesen ${ }^{3}$
}

${ }^{1}$ NCC Construction Denmark A/S and Technical University of Denmark, Management Engineering, Operations Man-
agement, Produktionstorvet, Building 426, room 042, 2800 Kgs. Lyngby, Denmark; ${ }^{2}$ Technical University of Denmark,
Management Engineering, Operations Management, Produktionstorvet, Building 426, room 013, 2800 Kgs. Lyngby,
Denmark; ${ }^{3}$ Technical University of Denmark, Management Engineering, Planning and Management of the Built Envi-
ronment, Produktionstorvet, Building 424, room 124, 2800 Kgs. Lyngby, Denmark

\begin{abstract}
The aim of this research is to discover how configuration systems can support a product's design process when a high degree of variation is required and a very open or endless space exists for possible configurations. The article is based on an industrial case involving a firm that wishes to offer a bathroom configurator to architects. The aim of the configurator is to help architects design a bathroom according to relevent requirements and norms. In offering the configurator, the firm aims to enable a design that can be coordinated with a prefabricated installation shaft sold by the firm, and also to create customer leads. Four scenarios are developed for how design can be supported by four different types of configuration technologies. The four scenarios are evaluated in relation to a number of functional and technical requirements. The scenarios indicate that a good and varied range of opportunities exist for using configuration systems in the construction industry. They also show that it can be done without fundamentally changing the present process.
\end{abstract}

Keywords: Configuration, expert systems, prefabrication, regulations, handicapped persons, installation, sanitary engineering, shafts.

\section{INTRODUCTION}

The construction industry has not achieved the same productivity gains as other industries producing physical products. This applies to both the price per square foot and quality and may be related to the building production method, which is characterized today by unique, one-of-a-kind projects [1]. The industry's companies focus on individual projects and not on a range of products to offer to the market that they could specialize in producing.

Other industries have achieved significant efficiency improvements by offering customized products that are not designed and produced as unique products, but rather selected and composed within a predefined range through mass customization, product architecture, modularization and configuration [2-5]. This method differs from traditional mass production in that the customer, within a range of prespecified boundaries, has the opportunity to individualize the product he receives, and still at a price that is closer to the price of mass-produced products than the price of individually manufactured products [6-11].

Mass-produced standard houses that were especially popular in the 1960s and 1970s are no longer as attractive and appealing, either to the customers or to the authorities issuing licenses to build. This is the case despite the fact that

*Address correspondence to this author at the NCC Construction Denmark A/S and Technical University of Denmark, Management Engineering, Operations Management, Produktionstorvet, Building 426, room 042, 2800 Kgs. Lyngby, Denmark; Tel: +45 24887859; Email: aku@ncc.dk this type of house can offer significant efficiency improvements. Thus, a need exists to individualize houses and at the same time use well known solutions and methods. This is where mass customization and system deliveries become relevant for the construction industry [12].

To achieve the goal of mass-customization, it is important to establish a strong and fixed connection between the product and the business processes, including the design process. To make and standardize such a connection, some companies have implemented a configuration system [13], which is often used by either a sales team or the customer. A construction project, however, is often organized in a much more fragmented way, where the customer, designers, contractors and the supplier of the system deliveries are located in different companies. This fragmentation creates a number of new issues in relation to the implementation of configuration systems.

This article describes and evaluates four scenarios for configuration systems, which a company can develop and offer to the market. In a larger context, the scenarios can be seen as examples of the opportunities that exist to use configurable system products in the construction industry.

\subsection{The Case}

One of the largest contractors in Denmark, along with several partners, has developed a prefabricated and configurable installation shaft $[14,15]$. See (Fig. 1). The shaft contains the vertical pipes for warm and cold water, heating, drains and ventilation, as well as cable trays for electrical installations, and is mainly for use in apartment buildings. 


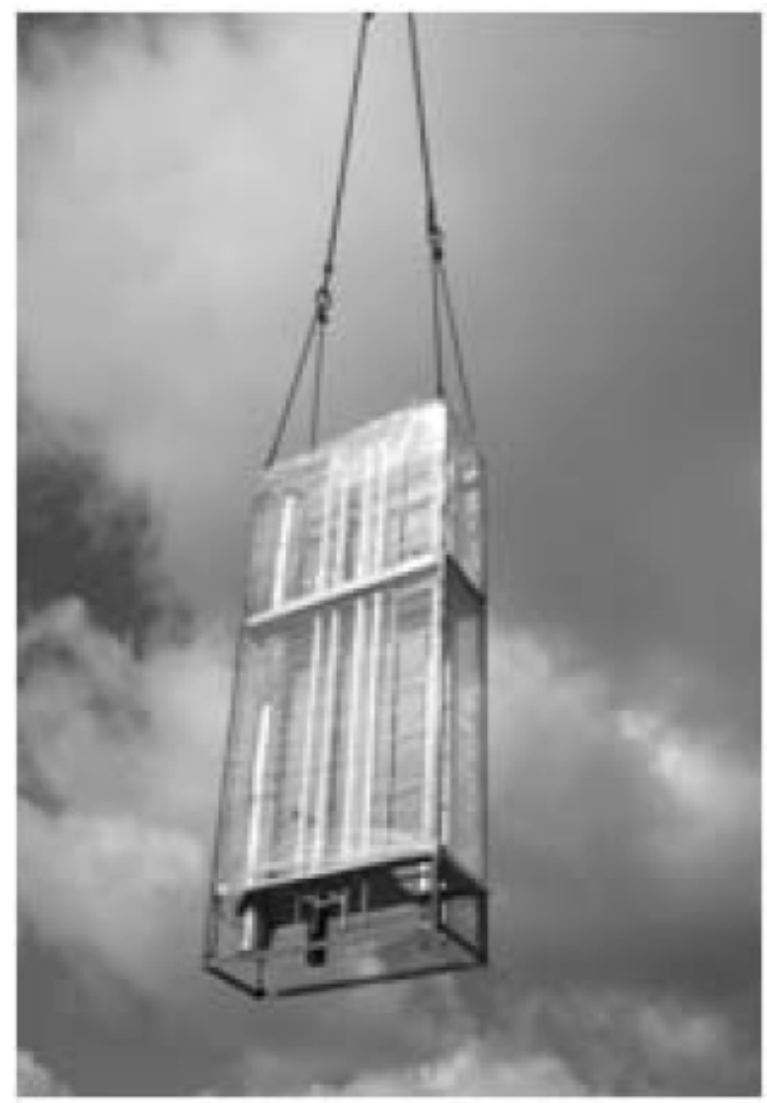

Fig. (1). The prefabricated shaft.

Today, the company supplies this prefabricated installation shaft to construction projects both inside and outside the company.

To realize the full benefit offered by the prefabricated shaft, and to ensure that the construction process is ready for such a system, it is important that it is incorporated early in the design phase, preferably already when the architect designs the floor plan, especially for the bathroom and of course for the shaft. To support this incorporation, the company wants to make a configuration system available to architects so that they know about the shaft early in the design phase. The company wants to make the configurator available on the Internet in order to provide easy access. Since it is often neither possible nor desirable to force the architect to use such a configurator, the configuration system should offer the architect some benefits in relation to the traditional way of designing bathrooms and shafts; it is these benefits that must motivate the architect to use the configuration system and thus provide the basis for the subsequent use of the prefabricated installation shaft.

In order to achieve its goals, which include making the configuration system attractive to architects, the company has developed a list of wishes for the configuration system that have resulted in a number of requirements for the configuration technology. Some of these differ from what is typical in developing configuration systems.

The areas where the requirements differ are as follows:

- The users of the configuration system, i.e. those who produce the part of the configured product that relates to the bathroom, come from other companies than the company providing the configuration system.

- The user must not actually or emotionally experience that his creativity is being reduced.

- The part of the user community that normally uses architectural 3D CAD / BIM software expects a nice graphic user interface, where substantial parts of the interaction take place by dragging elements to the desired location rather than by entering values and selecting from drop down lists.

- The solution space is very open, where only a few things are allowed, or only allowed, under certain conditions.

- It must be possible to transfer the configured result to the software that is being used to carry out the remaining part of the building design.

\section{PROBLEM FORMULATION}

A number of different examples exist of the use of configuration systems for configuration of construction products. Such examples are kitchen manufacturer HTH [16] and furniture manufacturer IKEA [17]. Studies of the use of mass customization and product configuration in other industries for example, the mechanics and electronics industries - show that significant benefits can be obtained in terms of faster provision, reduced errors and well-defined product families [18-20]. Also within the construction industry, the assumption is that considerable gains can be obtained by using these methods; however, the traditional way of organizing construction in Denmark creates a number of barriers to using these methods.

One of the important barriers in relation to the use of configuration systems is that many products in the construction industry have a large variety of dimensions, which results in an endless number of possible combinations and designs - an endless solution space. Traditional configuration systems require a fixed solution space, not only in mathematical terms but also in terms of practical management. When such a solution space is defined, the configurations system can help the user find a legal and optimal solution in accordance with a number of constrains.

Another barrier is that the building design is created independently of the producers - i.e. the material suppliers and the contractors - just those parties that in other industries traditionally describe the product families and make the configuration systems available.

In this article, we set up scenarios to show the possibilities that different technologies, with different functionality, offer for applying configuration in the design of a product with endless solution spaces - using the design of bathrooms and installation shafts as a case. The scenarios are subsequently evaluated in relation to a number of functional user requirements, as well as an economic assessment of the benefits, costs and risks of realizing the scenarios.

\section{THEORIES AND METHODS}

\subsection{Configuration and Mass Customization}

Mass Customization - the basic principle of this concept is to create value for the customer by adapting a product to 
his specific needs. The customer thus perceives that he is receiving a tailor-made product. From the manufacturer's point of view, the product is manufactured from uniform parts that can be produced using a specialized production apparatus; from the customer's point of view, it is a unique product [20].

This means that the idea of customization is to develop a portfolio of products that enables the company to offer the customer what is perceived as a unique product that matches the individual customer's needs. At the same time, the products in the portfolio have a number of common features with respect to design, production and assembly/installation. This means that the products can be considered the same and are therefore easier to produce, assemble and install.

The concept of Mass Customization [21, 22] describes a tendency in which companies that have previously manufactured mass-produced and uniform products start to manufacture these products in a continually increasing number of variants, so as better to be able to fulfil their customers' requirements [7].

The Mass Customization concept is supported by the use of configurating systems, which to a greater or lesser extent can support the design and specification of a customized product. A clear and very use-oriented definition of what a configuration system is: "... a software system that creates, uses and maintains product models that allow complete definition of all possible product options and variations with a minimum of entries" [23, 24].

\subsection{Method Description}

Four scenarios are set up to show how the design process, as a small step towards mass customization, can be supported in whole or in part by configuration. The scenarios are created and evaluated through a four- step method, based on Action Research theory [25]. The four steps are Plan, Act, Observe and Reflect; they have not been conducted sequentially but partly parallel, in that the planning and reflection steps are combined. This is done to make sure that the four scenarios describe a wide range of technologies, and to provide the opportunity to reflect on and evaluate the scenarios in relation to each other. The scenarios are normative backcasting scenarios. According to P.W.F. van Notten [26], they can be described as simple scenarios, but with two out of three features that characterize a complex scenario: multiissue and multi-dimensional, but not multi-scale. The scenarios provide a snapshot of how the architect's design process can be supported by various configuration systems.

The scenarios consist of three parts, but they are not in all cases treated separately:

- Event: What kind of a configurator is introduced?

- How will it affect the situation?

- Why? (Who gets what out of it?)

The scenarios are evaluated both technically and functionally in relation to the established functional requirements, while also evaluating the costs and risks of realizing the scenarios described. The evaluations are carried out in accordance with the current state of configuration [27] and CAD/BIM technology.
A qualitative and relative assessment of the costs of establishing the configurators has been carried out. This assessment is partly based on a survey collecting experiences with development and use of configuration software in 18 companies. The assessment is partly based on experience with a demo-configurator developed as part of the present project. An assessment of risk involved in using the different kinds of technology has also been made. This assessment is based on the commonly used risk definition - Risk = probability $\mathrm{x}$ loss - which also forms the basis of Michel Bernaroch's method [28]. Michel Bernaroch has extended the method somewhat, providing the opportunity to create a relatively detailed quantitative estimate of risk. Each of the scenarios described in this article can be realized with very different project organizations and using different software. Thus, the assessment of risk in the scenarios is based on a qualitative assessment that focuses on describing the risk factors included in Michel Bernaroch's method.

These risk factors are:

- Platform volatility - will the software platform be stable or will it change often

- Application experience* - application experience of the development team

- Product complexity - how large and complex is the software platform

- Analyst complexity - how complex is the functionality of the application

- Programming-language experience* - how much experience does the development team have with the software platform.

*Experience is described based on existing scientific articles that describe the use of the technology in relation to the configuration. Experience is also described on the basis of whether it concerns a general technology that many vendors can work with or a narrow technology that very few vendors can work with, and not in relation to possible future organizations' experience with the programming language.

Each of the risk factors are evaluated, in accordance with Michel Bernaroch's methodology, on a scale of Low, Medium or High. To obtain a uniform scheme, this scale has also been used to evaluate the other parameters. The sole exception is costs, which are set up using an index.

To find the potential benefits, the configurator's ability to provide value to the company and the users (the architects), the scenarios are evaluated in relation to a number of possible benefits. The scenarios were presented to and evaluated by four architects in order to obtain their opinions about how the various scenarios support their needs and requirements.

\section{IDENTIFICATION OF REQUIREMENTS, USERS AND SCENARIOS}

\subsection{Identification of Requirements}

The main value for the company is the ability to provide the company with leads on potential customers and projects. To achieve this, the company has set up a number of subbenefits, which are formulated here as functional requirements. They are: 
1. Provide shaft dimensions

The configuration system must provide the dimensions of the shaft. This will prevent them from being changed later, as happens in traditional projects when the engineer is involved in the process of making his shaft design.

2. Regulatory requirement control

The configuration system must ensure that the design meets the required standards and regulatory requirements $-\mathrm{BR}, \mathrm{SBI}$ and DS.

3. Freedom in design

The configuration system must allow the architect to design exactly the bathroom he wants within the framework of the chosen standards and regulatory requirements.

4. Handling of the bathroom's key elements

The configuration system must be able to handle the main elements of the bathroom: Door, shower, washbasin and toilet.

5. Ability to transfer results

The design must be able to be used in the further design process, i.e. be transferable to the other programmes that are used or be carried out directly in them.

6. Smooth, elegant and intuitive user interface

The configurator must be "smooth and elegant", intuitive, and must inspire the creative process.

\section{Provide leads to the company}

The configurator must provide the company with leads on potential customers, including information on projects where the shaft can be used.

\subsection{Identification of Users}

Users are identified by analyzing the process and the roles of a typical building project in the Danish construction industry. This analysis is combined with other studies that show, like projects in other industries, that at the start of a project there is a great need and the freedom to make decisions, which are expensive to change later in the process. See (Fig. 2).

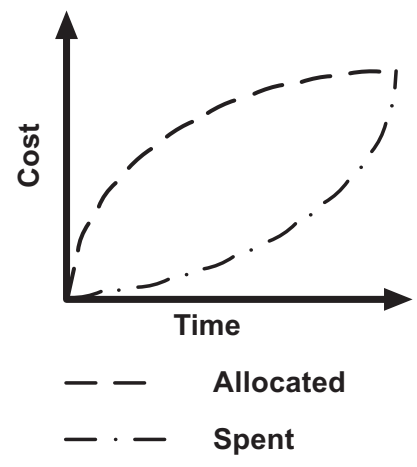

Fig. (2). Costs are allocated in the early stages.

The first actor that the configurator can target is the architect, who more or less makes decisions together with the developer [29] in the early stages.
If, instead, the configurator were targeted, the next step in the process - i.e. the shaft design by the engineers - would require the engineers, when using the configurator, to coordinate with the design the architect has already made. Such coordination can cause redesign for both the engineer and the architect, just as in the present process, where we have no configuration system.

The design and construction process of today is very fragmented. The design is made by a number of engineering and architectural firms, and construction is performed by a series of main contractors and/or subcontractors that buy their materials from a variety of suppliers. Simultaneously, throughout the process, the project organization develops gradually. This creates a situation where those who make the design do not know who will subsequently do the construction and what products they will use. Conversely, those who do the building often have very limited opportunities to influence the design to suit their preferred method and materials.

If we wish to introduce configuration systems into the current construction process and organization, it is necessary to make the configuration system available early in the process, even if those who would provide the configuration system later, or maybe never, are part of the process.

The developer or building owner is the first part in a construction project, and examples do exist where configuration systems are provided to the developer or building owner based either on a top-down approach, where the entire building is configured (e.g. core house [30]), or a bottom-up approach, where individual elements of the building are configured (e.g. HTH kitchen configurator [16]). In these examples, customers are allowed to configure e.g. a kitchen. These kinds of configuration systems typically target private customers who are interested in a house or a kitchen for a family. Professional developers usually have an architect clarify their needs and make the detailed designs (e.g. for kitchens or bathrooms). The case firm's shaft mainly targets apartment buildings, so the customers are normally professionals who have an architect to make the design.

\subsection{Identification of Scenarios}

The scenarios are based on the knowledge that the best way to achieve the aim to prepare a design for the case firm's shaft is to use a configuration system that can incorporate it already in the early stages of the architect's design process.

Each scenario is based on different software support, ranging from the traditional configuration software, e.g. in ERP systems like SAP or Microsoft Axapta, or specialized configurators like Tacton Configurator or Configit Product Modeler, to 3D CAD/BIM software like Auto Desk's Revit or Graphisoft's ArchiCAD, to game technology like Adobe ${ }^{\circledR}$ Flash (see [31]), which provides another example of the development and use of graphic configuration systems. Choice of software type is based on the software types used by members of the Association for Product Modelling in Denmark [32] and an investigation of the benefits that firms have received from using configuration [33]. 
The scenarios are created by combining the design processes and roles with the various software options available considering software that is normally used in the construction industry and which to a greater or lesser degree is targeted configuration in general. Then, the scenarios are examined to find out how they can fit into the process, and what part of the process they can support.

We have identified four scenarios, each of which can be realized by using different technologies:

1. Rule configurator: based on normal programming and database technology, like SQL and .NET.

2. Archetype configurator: based on expert systems, like Tacton Configurator or Configit Product Modeler.

3. Graphic configurator: based on game technology, like Adobe ${ }^{\circledR}$ Flash.

4. CAD configurator: based on BIM technology, such as Autodesk Revit or ArchiCAD.

As part of the scenarios, we have carried out a functional and technical assessment.

\section{SCENARIOS}

\subsection{Rule Configurator}

This configuration system is used to collect and present the norms and standards to be observed when designing a bathroom. The output from this configuration system is not the bathroom design, but rather part of the foundation to be used when building the design.

In Denmark, four sets of rules and norms provide a minimum framework for how bathrooms can be arranged:

\section{Building Code BR08 (224 Pages)}

The Danish building code comprises a set of rules that specify the minimum acceptable level for the function and technical solutions for new houses [34].

\section{SBI Guidelines 216 (387 Pages)}

"Guidelines on Building Regulations 2008, SBI instruction 216. SBI recommendations 216 on BR08 present background and interpretations which build bridges from the Building Regulations' general rules for constructability solutions. Authorization includes the building code text and the same structure. Instructions have also references to standards, instructions and other background material that is further explained with concrete solutions" [35].

\section{SBI Guidelines 222 (70 Pages)}

The instructions justify and recommend a series of accessibility requirements for ordinary dwellings, based on Building Regulations 2008 (BR08). The recommended goal is typically greater than the regulatory requirements. The recommendations are essential, both for planning and designing new buildings and for renovations and additions to the existing housing stock [36].

\section{Danish Standard 3028:2001 (184 Pages)}

The standard sets out requirements for buildings and facilities and their accessibility, which must be available to everyone, including people with disabilities [37].
Each of these regulations provides rules for three categories of bathroom users:_Normal, Disabled - self-reliant, and Disabled - intensive care.

Within each of the three categories, each set of rules and norms has from 3-18 different rules that affect the layout of the bathroom. Some of the rules are identical and others vary across regulatory and/or user category. For the architect, this means that in order to design according to all four sets of rules/norms in all three user categories, he must deal with a total of 31 rules, of which only one sub-set must be followed. The rules are today described in four books, a total of 865 pages.

One way a configuration system could work is by letting the user specify two things: 1) the user category and 2) the rules and standards to be followed (see Fig. 3). With this input, the configuration system can generate a list of rules for the design. Depending on the post-processing, layout and number of illustrations required, such a list will amount to about 1-3 A4 pages.

The output can be post-processed in a number of more or less intelligent ways:

- Simple extraction: Four sets of rules and norms are filtered so the output consists of only the relevant parts from each set. No editing or coordination between the various rule/norm sets is made.

- Edited extraction: The output is filtered and redundant rules across the rule/norm sets are removed, especially between BR08 and SBI 216, since SBI 216 is an interpretation of the requirements in BR08. Also, the output includes the most appropriate illustrations of the various norms and regulations.

- Analytical extraction: In addition to clearing the output of redundant requirements, the list is also interpreted so that it only shows the most demanding requirements within a given area.

The functionality of this configuration system is also integrated into the configuration systems described in the other scenarios, but the output in the other scenarios is not necessarily communicated to the user. Instead, it is used internally, in the configurator, to adjust the set of regulations.

The output from the configurator consists of an extraction and reproduction of rules and norms to which the supplier of the configuration system does not have copyright. This issue must be resolved before developing such a configuration system. How the copyright issue should be handled in practice is outside the scope of this research.

\subsubsection{Evaluation}

\section{Functional}

This configuration system helps the architect find the rules he needs. Ehe architect does not need to remember the rules or look them up in up to four different books and run the risk of either finding the wrong rules or missing some of them. The architect can thus reduce the time spent on making the design, but the main potential probably lies in fewer errors in the design and fewer designs rejected by the authorities, which results in a reduction both resources and calendar time used. At the same time, the architect gains the 


\section{Product configuration}

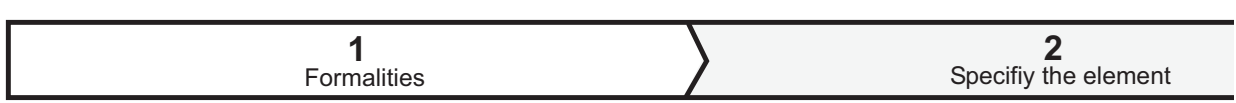

Bathroom

What rules and standards must be followed?

$\begin{array}{ll}\text { Categoric of bathroom users } & \text { General (Click to confirm) } \\ & \text { Disabled - self reliant } \\ \text { Disabled - intensive care } \\ \text { Building code BR08 } & \text { Yes } \\ \text { SBi guidelines } & \text { Yes (Click to confirm) } \\ \text { Danish Standard } & \text { No (Click to confirm) }\end{array}$

Fig. (3). Example of rules selection.

opportunity to focus fully on the architectural values in the bathroom design.

In accordance with the case company's aims, this configuration creates value by linking the company providing the configuration system and the architect who uses it. The company can potentially use this link to engage in dialogue with the architect to try to influence him to prepare his design for use of the company's shaft. This dialogue, together with concrete descriptions of how the architect ensures such preparation, lies outside this configuration system and basically consists of purely manual processes.

\section{$\underline{\text { Technical }}$}

The solution space within which this configuration system must work contains a finite number of solutions and is thereby a finite solution space, within which the configuration system must find the relevant rules.

The input to this configuration system could be in the form of a series of choices, where the user chooses among the options offered within the rules, standards and user category via e.g. drop-down menus, check marks or radio buttons.

As previously described, the result must be edited somewhat before it is returned to the user. Apart from the graphic part, this editing is mostly carried out according to rules that consist of mathematical/logical operations, such as: if $a>b$ then output $=$ a. But some rules and norms require a subjective interpretation. If they must also be interpreted before being returned to the user, the configuration system must contain a strictly logical interpretation of the rules and standard requirements.

The above requirements can be met by using ordinary programming and database technology, such as Microsoft .NET and SQL. This kind of software is designed to show its results on the internet, using e.g. aspx.

\section{Price and Risk in the Development Project}

This configuration system contains a very limited number of rules and attributes. This, combined with the opportunity to use well known and widely used technologies, makes the risk associated with developing a configurator relatively small from a purely technical viewpoint.

The price for the development of such a configuration system is considered to be relatively low, but it obviously depends on the type and amount of graphics to be included in the user interface, among other factors. This assessment does not include any costs associated with the management of copyrights.

\subsection{Archetype Configurator}

This scenario is based on a finite number of bathroom layouts that the architect can choose among. These layouts are made by splitting the bathroom into 12 sections, in which each of the four main elements (toilet, washbasin, shower and door) can be placed (see Fig. 4). This gives $12 * 11 * 10$ $* 9=11,880$ possible combinations, but not all of them are legal. Because the numbers are not explicit, the figure can be rotated without changing the solution, which provides a logical definition of the solution space, reduced to a quarter, which gives 2970 possible solutions.

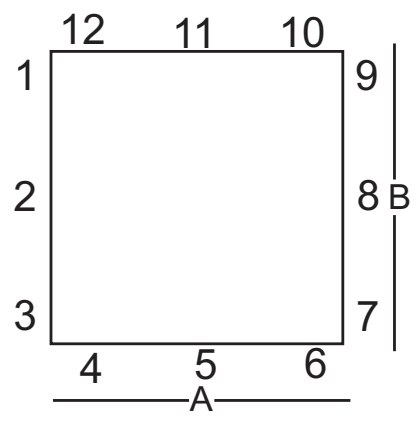

Fig. (4). The 12 sections of the bathroom.

In principle, the configuration can be carried out by showing the user a large overview of all possible combinations for location of the bathroom components - toilet, washbasin, shower and door - with the desired variations of individual components, such as shower or tub. The architect 
can then choose the design he wants and thereby be sure that it is legal. The configuration system then calculates the minimum dimensions, $\mathrm{A}$ and $\mathrm{B}$, for the desired set of rules/norms and user category. But a table with up to 2970 possible combinations, plus the desired variants, cannot work in practice. Instead, the architect can arrive at a solution more easily by allowing him to choose the location of each component separately.

Example: A bathroom is to be designed according to the BR and DS rule/norm sets for the user category "disabled self-reliant". If the toilet is placed in section 2 , it will be possible to place the washbasin in either section $1,3,4$ or 12 (see Fig. 5).

The configuration system is then able to calculate the minimum permissible bathroom dimensions, A and B (see Fig. 6). The architect can then enlarge the bathroom to the size he wants. It is also possible to show the user a nonscaled diagram of the bathroom layout with the selected components and their locations. Using Tacton's technology, this drawing can be developed according to the same principle as the configuration, by making transparent images of the bathroom elements in their possible locations and then stacking the desired versions in order to generate an overall picture.

When the configuration is finished, it is also possible to send the parameters from the configuration system to a CAD program [31]. Tacton supports this, with e.g. SolidWorks, and can thereby obtain a 3D CAD model of the desired bathroom. This possibility supports the further use of the configuration, but does not support the actual configuration. This, combined with the lack of interfaces to CAD systems traditionally used in the construction industry, means that this option is not included in the scenario.

\subsubsection{Evaluation}

\section{Functional}

As a part of this research, a prototype configuration system was made of the type described here. The prototype was used to obtain knowledge about the possibilities and challenges of making a bathroom configurator. The research shows that this scenario does not allow the architect to design bathrooms that meet the required rules and norms within the desired user category. But it also shows that it is a very rigid process that only interacts with difficulty with the architect's creative process and can directly inhibit it.

Furthermore, the solution has the disadvantage that it only works with the minimum bathroom size. After the configuration, the bathroom must be extended to the desired size outside the configurator. This means that the larger the bath- room, the bigger the difference between the configured bathroom and the one actually wanted.

This type of configuration system provides the greatest value when used for minimum bathrooms, e.g. in public housing, where price and function are often the most important parameters.

The fact that the architect must change the configured design, if he does not want a minimum-size bathroom, means that this scenario is not able to provide the dimensions and location of the shaft.

\section{Technical}

The prototype shows that creating a configuration system of this type presents no significant challenge, and that it can be made with traditional configuration technology. The prototype is developed in Tacton Configurator. It is, however, a challenge to transfer the result from the configurator to the architect's CAD or BIM software. According to Tacton, the challenge is that it cannot export to CAD file formats like IFC or dwg, formats that are normally used for the exchange of building models in the construction industry.

Tacton has an integrated web-based user interface, which makes it possible to make the configuration system available on the Internet without having to involve further technology (see Tacton's homepage) [39]. The biggest technical challenge is thus to create a graphic interface that appeals to architects and supports their creative design process.

\section{Price and Risk in the Development Project}

The work with the demo configuration system developed in this project shows that technically it is not a big challenge to develop a configuration system of this type. The critical risk factors are thus the classic ones - to select the right supplier and to develop the right solution.

The demo configurator contained about 230 rules and attributes; therefore, the price of developing a professional version of such a configuration system is estimated to be low.

\subsection{Graphic Configurator}

This type of configuration system works in the way that the user defines a room in either $2 \mathrm{D}$ or $3 \mathrm{D}$, and then drags the desired elements from a library to place them inside the room. The element can be moved around in the room. When the user releases it, the configuration system validates the placement and indicates whether it is legal or not. If it is not legal, the configuration system indicates this by, e.g. making the element red and/or automatically moving the element to a legal place. Once the user has configured the bathroom, he

Location for the toilet
Location for the washbasin
Location for the door
Location for the bath

Fig. (5). Placement of the washbasin.

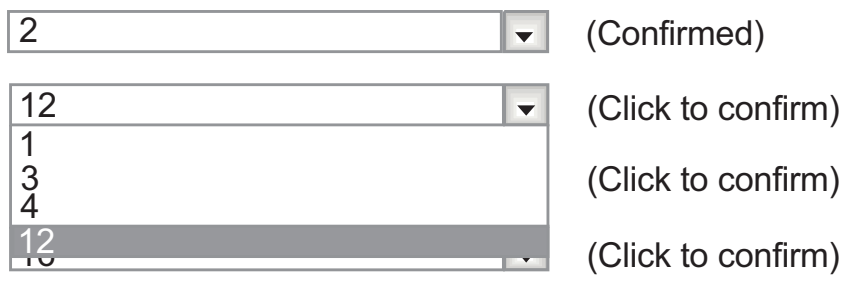


can download a 3D CAD or BIM file that can be used as the basis for design in the architect's own BIM or CAD system. In recent years, a number of graphic configuration systems like this have appeared. The world's largest furniture company, IKEA, and the Danish kitchen company, HTH, are examples of companies providing this kind of configuration system to their customers. Fig. (7) shows a screenshot from HTH's graphic kitchen configurator on HTH webshop [16].

In spite of the large number of graphic configuration systems, it has not been possible, during the literature study for this article, to find any scientific articles describing the behaviour and underlying form of reasoning of this type of configuration system. HTH's configurator uses Adobe ${ }^{\mathbb{B}}$ Flash technology, which is also used by other configuration systems as well as by many online games (see the onlineflashgames homepage [38]).

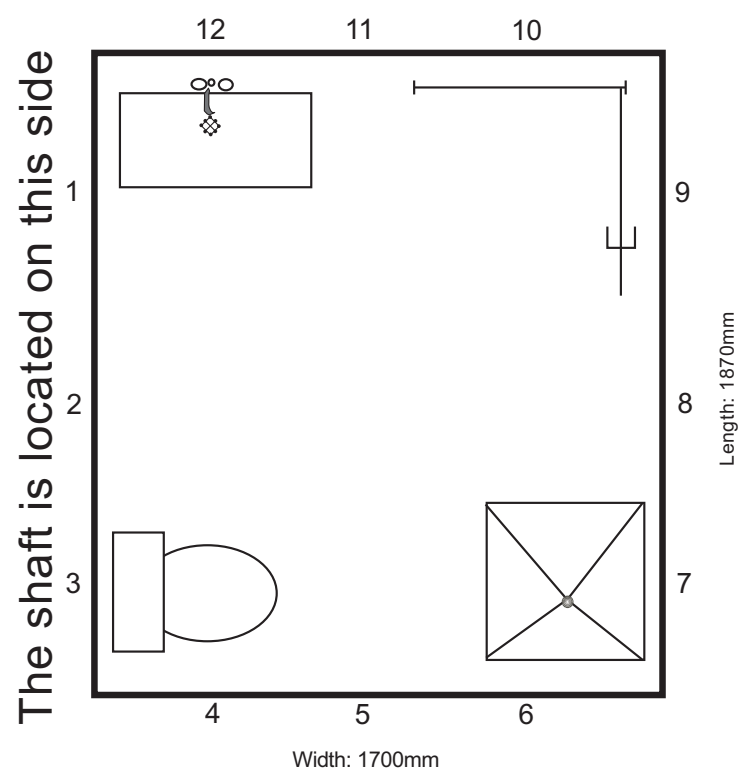

Fig. (6). Overall picture of the bathroom.

\subsubsection{Evaluation}

\section{Functional}

The use of this type of configuration systems is in many ways similar to that of a BIM system. It is often so simple that it can be used not only by professionals such as architects, but also by private customers without any specific professional prerequisites. Both HTH and IKEA target their configuration systems directly to their private customers.

Users of this type of configuration system can feel great freedom compared to other, more choice-based configuration systems. This does not necessarily mean that they can design bathrooms that could not have been designed using the configuration systems described in the other scenarios, but it is done here in a flexible way that supports the creative process.

\section{$\underline{\text { Technical }}$}

As mentioned, in the literature study for this article, no descriptions could be found of the technical possibilities and challenges of using the Flash technology to make graphic configuration systems. But the Flash technology is made to be used on the Internet and is therefore very suitable for this medium. Also, examples are seen of Flash-based configuration systems that can export BIM models, e.g. in IFC format (see boligtjek homepage [40]).

One configuration software producer states that in their solutions, they use technologies that are also used in the gaming industry. And in our graphic, spacious and rulebased world, a number of similarities seem to exist between configuration systems and games.

\section{Price and Risk in the Development Project}

Flash is a widely used technology, and in connection with the research for this article, we found a large number of more or less advanced configuration systems using this technology. Such widespread use means that there are also many vendors offering solutions based on this technology, which contributes to keeping both price and risk down. However, especially because the graphic user interface is relatively advanced, the price is also assessed to be relatively high. Also, the fact that it has not been possible to find scientific articles about use of the technology to develop configuration systems is interpreted to indicate that such an application is relatively new; therefore, the risk is assessed to be relatively high.

\subsection{CAD/BIM Object Configurator}

In the construction industry, which includes architects, we see an increasing use of $3 \mathrm{D}$, object-oriented CAD/BIM systems such as Revit and ArchiCAD. This type of system gives the architect freedom to design what he wants within the technical framework of the system. These systems are not traditional configuration systems, but rather advanced drawing or modelling tools.

The models made in the BIM systems are not traditional CAD drawings with lines in two or three dimensions. These models are composed of parametric 3D objects representing the parts of the building. Some of these objects can be perceived as small configurators that can adapt the object by using a variety of input data. The objects are programmed in a language that is specific to each BIM system. For instance, objects in ArchiCAD are programmed in Geometric Description Language, GDL. Through their various languages, BIM systems make it possible to build large or small configurable objects [41, 42].

Because the languages are different, objects must be developed to target each of the $\mathrm{CAD} / \mathrm{BIM}$ systems that the case company wants to support. This factor presses the price of this solution up; however, it also makes it possible to adapt the project size, e.g. by choosing to develop items for only one or two systems.

A number of material product suppliers in the construction industry have established object libraries, which contain models of their products (see www.3dbyggeri.dk). By making these libraries available to architects, the products are "automatically" built into the models for the building that will subsequently be built.

The company can similarly establish and make a bathroom and shaft object available to architects, who can then 


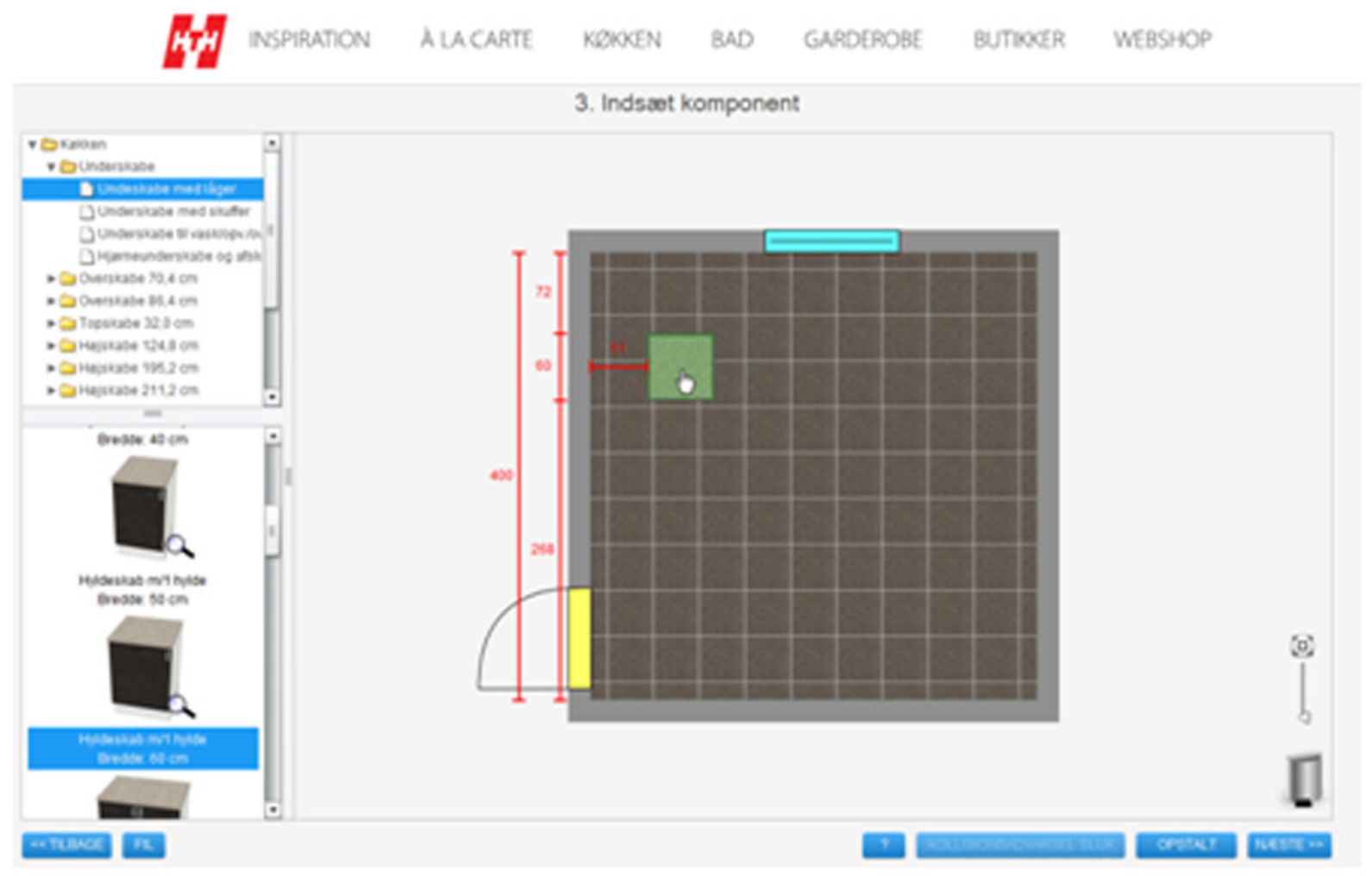

Fig. (7). Graphic configurator from HTH webshop [16].

download the object, include it in their own object library, and use it as an integral part of their own CAD systems. Thus, architects are provided with a guide for designing legal bathrooms and shafts with the right dimensions and prepared for the company's prefabricated shaft module.

\subsubsection{Evaluation}

\section{Functional}

The solution has the major disadvantage of requiring the architect to have and use one of the BIM systems that the company chooses on his own computer. No precise estimate is found of how many architects are using BIM software, but at present, since this technology is spreading rapidly, we do not consider this a major barrier. However, it would not be possible for an architect who uses e.g. a traditional 2D CAD program to use the configurator. The fact that the solution runs on the user's own computer, in a system used normally, provides great benefits:

- The user will not need to acquire experience with a new system in order to use the configurator.

- The user has the solution "at hand" as an integral part of the system they already work with.

- After the configuration is complete, the results will not have to be transferred to the user's own system.

A disadvantage of this solution is that it cannot be stored and run centrally from the provider's server. It runs on the individual user's computer. This means that the user must verify that the object being used is the latest version. If the user does not make such verification, it is not certain that the relevant object follows the latest rules and standards.
Even though the bathroom is configured directly in the BIM system and does not subsequently have to be transferred from one system to another, this does not mean that finishing is not required. For example, the angle of the floor surfaces must be defined, along with lighting, mirror and other elements that the configurator does not handle.

\section{Technical}

Since each of the major software vendors uses its own language to develop objects, separate objects must be developed and maintained for each system. If the company wants to make the solution available to ArchiCAD users, GDL Objects (.GDL) must be developed. Likewise, Families (.rfa) must be developed, if the company wants to make the solution available for Revit users.

The solution is only available on the Internet insofar as users can download the desired objects from a webpage. The subsequent configuration is done in the user's own BIM program. This makes the solution simple from the company's point of view, since the code is not run on the company's servers.

In their basic structure, BIM objects are not prepared to work with multiple rule sets. If the company wants to cover all types of bathrooms and all combinations of rules, it would probably be best to make an object for each combination. If all combinations were to be covered, up to 30 different objects would have to be developed and maintained.

With respect to the company's ability to gain knowledge about potential customers and projects, this type of configurator is very unfortunate. Once the client has downloaded the object, the company would not receive any information 
about when, who and for what project the configurator is used.

\section{Price and Risk in the Development Project}

In this solution, several factors that affect price and risk pull in opposite directions. The fact that the configuration system runs on the users' own computers means there are no costs for hosting and managing an online configuration system. The fact that objects must be developed for each of the various BIM systems that the company wishes to support pulls in the opposite direction. Each of these systems has its own way of making objects, along with its own programming language, which means duplication of the work. It also requires that the development organization can handle all the selected technologies. Overall, the price for this solution is assessed to be in the middle.

In relation to risk, the solution is based on well-known and widely used technologies, which should keep risk down. It is also based on technologies that cannot be controlled, however, and the risk exists that they can be changed and the company forced to make updates at times over which it has no control.

\section{EVALUATION OF THE SCENARIOS}

The four scenarios are assessed with respect to fulfilment of the company's functional requirements, as well as in relation to cost and risk. Cost is calculated as an index based solely on development cost. Scenario 2, for which we have made a demo configuration system, is set to index 100 .
Table 1 shows that among the four scenarios, the most benefits are obtained by realizing scenario 3 . Scenario 4 is good when measured on the value to architects, but loses on the possibility to generate leads for the company. We have not scored the various requirements, but the lack of leads is so critical that this scenario cannot be realized with success. Scenario 3 is relatively the most expensive scenario to realize, and it is associated with high risk. But because the Flash technology is widespread, it must be possible to clarify this risk very early in the project process and thereby deal with it.

In the less productive end, we find scenarios 1 and 2 . Scenario 2 has the most gains of the two, but it also results in a quite locked design process that can discourage architects from using the configurator. At worst, it could create a bad atmosphere in the construction industry in relation to the use of configuration in general.

\section{CONCLUSION AND PERSPECTIVES}

In this study, four scenarios are evaluated in relation to how a firm can develop and make available a bathroom configurator. The aim is both to ensure that the bathroom design is prepared so that it is compatible with the installation shaft sold by the case company, and to create contact with potential customers. Bathrooms differ from products for which configuration systems have been traditionally used in that they have a large or infinite solution space, which traditional technologies, such as the Tacton configurator, cannot manage directly. In order to manage such a solution space, the first two scenarios, which use traditional configuration tech-

Table 1. Evaluation of the Scenarios

\begin{tabular}{|c|c|c|c|c|}
\hline & $\begin{array}{c}\text { Scenario } 1 \\
\text { Rule configurator }\end{array}$ & $\begin{array}{c}\text { Scenario } 2 \\
\text { Archetypes }\end{array}$ & $\begin{array}{l}\text { Scenario } 3 \\
\text { Graphic }\end{array}$ & $\begin{array}{l}\text { Scenario } 4 \\
\text { BIM/CAD }\end{array}$ \\
\hline \multicolumn{5}{|c|}{ Functional requirements } \\
\hline Provide shaft dimensions & Low & Low & Low & High \\
\hline Control of regulatory requirements & High & Medium & High & High \\
\hline Handling of the bathroom's key elements & Low & Medium & High & High \\
\hline Ability to transfer results & Low & Low & Low & High \\
\hline Smooth, elegant and intuitive user interface & High & Medium & High & High \\
\hline Leads to the company & Medium & High & High & Low \\
\hline Application experience & High & Low & High & Medium \\
\hline Product complexity & Low & Low & Medium & High \\
\hline Analyst complexity & Low & Low & High & High \\
\hline Programming-language experience & High & High & Low & Medium \\
\hline \multicolumn{5}{|c|}{ Development cost } \\
\hline Development cost index & 38 & 100 & 223 & 169 \\
\hline
\end{tabular}


nology, operate with configuring a basis that can be used for the final design, which is then carried out outside the configuration system.

The great advantage of the CAD configurator is that the configuration is done directly on the user's own software, which means that the user works in a known environment, and that afterwards he does not have to transfer the configuration, since it immediately becomes part of the total design. This solution has the disadvantage that it requires the user to have one of the software packages that the case company chooses to support. The solution fails however in relation to an even more important requirement for the case company: it cannot provide customer leads. Therefore, an essential benefit for the company is lost. In addition, the solution would demand a relatively large development effort to support the different platforms.

The fourth scenario uses a technology that is also used in online computer games as well as in developing online configuration systems. This technology differs from traditional configuration technology in that it operates with an open solution space, which allows the architect great freedom in the design. It is also based on a visual presentation that makes it possible to provide a smooth, elegant and intuitive user interface. The scenario has however the disadvantage that in spite of an intense search, it has not been possible to find scientific articles describing how the technology works or providing other examples of the technology's application to configuration systems. This is thus an area that needs further research, which is also the case for the whole area having to do with configuring products with very large or infinite solution spaces.

All four scenarios describe the current situation where the design process has not been fundamentally changed. It is still the architect who makes the bathroom layout, without specific knowledge about how the bathroom and shaft will arrive at their final design, how they will be constructed, or by whom. Only the actual design process is modified, and only this process is directly supported by the configuration system. The scenarios indicate that, to start with, good and varied opportunities exist for using configuration systems in the construction industry. They also show that it can be done without fundamentally changing the present process.

They also indicate, however, that the overall setup where the configuration system is provided by a party that enters the process much later, or maybe never - creates problems. One of the big challenges is to convince the architect of the advantages of using the configurator rather than maintaining his current process. It is not enough that the configuration system is an advantage for the overall construction process. It must also be so for the architect. This makes great demands on the configurator, both in terms of function, user interface and solution space. The configurator must not exclude solutions that the architect wants, even if these solutions are considered a disadvantage when evaluated in relation to the total building process. It is beyond the scope this article to assess whether this means that the construction processes and basic organization need to be changed in order to make it practicable and profitable to develop configuration systems.

\section{CONFLICT OF INTEREST}

The author(s) confirm that this article content has no conflicts of interest.

\section{ACKNOWLEDGEMENT}

Declared none.

\section{REFERENCES}

[1] C. Thuesen, and L. Hvam, "Efficient on-site construction: learning points from a German platform for housing", Construction innovation: Information, Process, Management, vol. 11, pp. 338$355,2011$.

[2] L. Hvam, "The rulers factory - a tool for learning product modelling techniques", Computers and Industrial Engineering, vol. 35, pp. 29-32, 1998.

[3] A. Joneja, and N. K. S. Nevilli, "Automated configuration of parametric feeding tools for mass customization", Computers and Industrial Engineering, vol. 35, pp. 463-466, 1998.

[4] V.B. Kreng, and T. Lee, "Modular product design with grouping genetic algorithm-a case study", Computers and Industrial Engineering, vol. 46, pp. 443-460, 2004.

[5] J. Jiao, M.M. Tseng, M. Mitchell, V.G. Vincent, and F. Lin, "Product family modelling for mass customization", Computers and Industrial Engineering, vol. 35, pp. 495-498, 1998.

[6] U. Harlov, Developing Product Families Based on Architectures, Denmark: Department of Mechanical Engineering, Technical University of Denmark, 2006.

[7] N.H. Mortensen, L. Hvam, P. Boelskifte, C. Lindschou, S, Frobenius, and A. Haug, "Making product customization profitable", International Journal of Industrial Engineering: Theory Applications and Practice, vol. 17, pp. 25-35, 2010.

[8] F. Salvador, P.M. De Holan, and F. Piller, "Cracking the code of mass customization”, MIT Sloan Management Review, vol. 50, pp. 71, 2009.

[9] L. Hvam, N. H. Mortensen, and J. Riis, "Product Customization", Berlin Heidelberg: Springer, 2008.

[10] L. Hvam, "A procedure for the application of product modeling", International Journal of Production Research, vol. 39, No. 5, pp. $873-885,2001$.

[11] A. Haug, L. Hvam, and N.H. Mortensen; "A layout technique for class diagrams to be used in product configuration projects", Computers in Industry, vol. 61, no. 5, pp. 409-418, 2010.

[12] D. Benros, and J. P. Duarte, "An integrated system for providing mass customized housing", Automation in Construction, vol. 18, pp. 310-320, 2009.

[13] Q. Yanhong, and W. Guangxing, "Product configuration based on modular product family modelling", Journal of Computational Information Systems, vol. 6, no. 7, pp. 2321-2331, 2010.

[14] Byggeriets Innovation. (2008). På vej mod fremtidens skakt, Byggeriets Innovation, Copenhagen. [Online] Available:

http://www.ncc.dk/skakt (in Danish).

[15] C. Thuesen, and L. Hvam, "Rethinking the business model in construction by the use of off-site-'system deliverance' - The shaft project", Journal of Architectural Engineering, 2012.

[16] HTH, HTH webshop, [online] September 2013, http://webshop.hth.dk (Accessed: September 15, 2013).

[17] IKEA, IKEA kitchenplanner, [online] September 15, 2013, http://kitchenplanner.ikea.com/US/UI/Pages/VPUI.htm (Accessed: September 15, 2013).

[18] L. Hvam, "Mass customization in the electronics industry - based on modular products and product configuration", Journal of Mass Customization, vol. 1, pp. 410-426, 2006.

[19] C. Forza, and F. Salvador, "Managing for variety in the order acquisition and fulfillment process: The contribution of product configuration systems", International Journal of Production Economics, vol. 76, pp. 87-98, 2002.

[20] M. M. Tseng, and J. Jiao, "Case-based evolutionary design for mass customization", Computers \& Industrial Engineering, vol. 33, p. 319, 1997.

[21] J. Pine, "Mass Customization - The New Frontiers in Business Competition”, Boston: Harvard Business School Press, 1999. 
[22] M. M. Tseng, and F. T. Piller, "The Customer Centric Enterprise", Springer-Verlag, Berlin Heidelberg, 2003.

[23] [Bourke, 1998] R. Bourke, "Configurators, a status report", Apics The Performance Advantage, vol. 8, no. 5, pp. 42-45, 1998.

[24] N. H. Mortensen, U. Harlou, L. Hvam, and A. Haug, "Improving decision making in the early phases of configuration projects", The International Journal of Industrial Engineering, vol. 18, pp. 452461, 2011.

[25] R. O'Brien, "An Overview of the Methodological Approach for Action Research". Faculty of Information Studies, University of Toronto, 2012. [Online] http://www.web.ca/robrien/papers/ arfinal.html

[26] P.W.F. Notten, J. Rotmans, M.B.A. van Asselt, and D. S. Rothman, "An updated scenario typology", Futures, vol. 35, pp. 423-443, 2003.

[27] A. Haug, L. Hvam, and N. H. Mortensen, "Definition and evaluation of product configurator strategies", Computers in Industry, vol. 63, pp. 471-481, 2012.

[28] M. Benaroch, and A. Appari, "Financial pricing of software development risk factors", IEEE Software September/ October, pp. 6573, 2010.

[29] FRI, PLR, Danske ARK, "Description of services for building and planning 2009", 2012 [Online]http://www.danskeark.dk/ Medlemsservice/Raadgiverjura/Aftalegrundlag/Ydelsesbeskrivelser/Bygger i-og-planlaegning.aspx

[30] A. Kern-Haus, [online] September 23, 2011, http://www.hauskonfigurator.de (Accessed: September 23, 2011).

[31] L. Hvam, and K. Ladeby, "An approach for the development of visual configuration systems", Computers \& Industrial Engineering, vol. 53, pp. 401-419, 2007.
[32] DTU, Productmodels, [online] September 15, 2013, http://www. productmodels.com

[33] A. Haug, L. Hvam, and N. H. Mortensen, "The impact of product configurators on lead times in Artificial Intelligence for Engineering Design", Analysis and Manufacturing, vol. 25, no. 2, pp. 197206, 2011.

[34] Byggecentrum, "Building Regulations 2008", Byggecentrum, Ballerup, 2008.

[35] SBI Guidelines 216, SBI Guidelines 216, Copenhagen: SBI, Aalborg University. 2008.

[36] L. Sigbrand, and H. P. Jensen, "Tilgængelige boliger", Copenhagen: Statens Byggeforsningsinstitut. 2008 (in Danish).

[37] Dansk Standard, "DS 3028:2001 - Tilgængelighed for Alle", Copenhagen: Dansk Standard, 2001 (in Danish).

[38] Onlineflashgames, ofg, [online] September 15, 2013, www. onlineflashgames.org (Accessed: September 15, 2013).

[39] Tacton, Demos, [online] September 15, 2013, http://www. tacton.com/design-automation/demos/\#fancy3556 (Accessed: September 15, 2013).

[40] Energistyrelsen, Boligtjek, [online] September 15, 2013, http://www.ens.dk/forbruger/boligtjek (Accessed: September 15, 2013).

[41] K. Agger, D. Kjøller, N. Knudsen, P. Kortegaard, and J. Skauge, "Produktkonfigurering i byggeriet", Aarhus: Aarhus School of Architecture. 2004 (in Danish)

[42] D. Nicholson-Cole, "The GDL Cookbook Verson 3", Nottingham: Marmalade Graphics, 2001

(C) Kudsk et al.; Licensee Bentham Open.

This is an open access article licensed under the terms of the Creative Commons Attribution Non-Commercial License (http://creativecommons.org/licenses/by-nc/3.0/) which permits unrestricted, non-commercial use, distribution and reproduction in any medium, provided the work is properly cited. 